\title{
Prevalence of Tinea Capitis among School Children in Nok Community of Kaduna State, Nigeria
}

\author{
Josephine Dogo, ${ }^{1}$ Seniyat Larai Afegbua, ${ }^{1}$ and Edward Christopher Dung ${ }^{2}$ \\ ${ }^{1}$ Department of Microbiology, Ahmadu Bello University, Zaria, Kaduna State, Nigeria \\ ${ }^{2}$ Department of Dermatophilosis, National Veterinary Research Institute, Vom, Plateau State, Nigeria \\ Correspondence should be addressed to Seniyat Larai Afegbua; seniyat.larai@gmail.com
}

Received 31 October 2015; Revised 28 March 2016; Accepted 17 April 2016

Academic Editor: Nongnuch Vanittanakom

Copyright (c) 2016 Josephine Dogo et al. This is an open access article distributed under the Creative Commons Attribution License, which permits unrestricted use, distribution, and reproduction in any medium, provided the original work is properly cited.

\begin{abstract}
In recent years, the prevalence of tinea capitis, an infection of the scalp by dermatophytes, has increased in children worldwide. This cross-sectional study was carried out to determine the prevalence and risk factor of tinea capitis among school children in Nok community of Kaduna State, Nigeria. A total of 100 children were screened and $45 \%$ were diagnosed to have tinea capitis after fungal culture and microscopy. The prevalence of tinea capitis among girls was higher (51.4\%) than that among boys (41.5\%) but not significantly different $(p=0.402)$. The prevalence with respect to age was lower for the age group 5-10 years $(42.6 \%)$ than that of $11-15$ years $(50 \%)$ but was not significantly different $(p=0.524)$. Trichophyton rubrum $(28.8 \%)$ and Microsporum canis $(22.7 \%)$ were the most prevalent dermatophytes isolated and the least were Trichophyton verrucosum (4.5\%) and Trichophyton tonsurans (4.5\%). There were $73.3 \%$ single infection while $26.7 \%$ had 2-4 dermatophytes of the genera Microsporum and Trichophyton. The predisposing factors with statistically significant association with tinea capitis were number of children in the family $(p=0.02)$ and sharing of the same bed $(p=0.002)$. This indicates the high tendencies of spread of tinea capitis through human-to-human mode of transmission and possible animal contact. Community health education on the cause, mode of transmission, prevention, and prompt treatment of tinea capitis is recommended.
\end{abstract}

\section{Introduction}

Dermatophytosis refers to mycotic infections caused by a group of fungi that possess the propensity to colonize and infect superficial layers of the skin, hair, or nails [1]. This group of fungi that invade keratinized tissue associated with the stratum corneum of the skin, hair, and nails on the living host are called dermatophytes [1]. Dermatophytosis is caused by a group of fungi with predilection to colonize and infect stratum corneum of the skin, hair, and nails on the living host. Such fungi referred to as dermatophytes include members of the genera Epidermophyton, Microsporum, and Trichophyton [1]. Dermatophytes are grouped as either anthropophilic (e.g., Trichophyton violaceum, Trichophyton rubrum, and Microsporum audouinii), geophilic (e.g., Microsporum gypseum and Microsporum nanum), or zoophilic (e.g., Trichophyton mentagrophytes, Trichophyton verrucosum, and Microsporum canis) based on their ecological niches; however, some dermatophytes are not strictly restricted to a particular ecological niche $[1,2]$.

Dermatophytosis has a worldwide distribution but is endemic to tropical regions as the growth of dermatophytes is facilitated by the warm and moist conditions $[1,3,4]$. Apart from climate, the variability in distribution of dermatophytes worldwide is attributed to other factors such as population migration patterns, lifestyle, primary host range, secondary host immunity, presence of immunodeficiency diseases, and patients attitude to prompt treatment following clinical presentation and standard of living $[1,5]$.

Depending on the body site affected, dermatophytosis may result in different clinical syndromes such as tinea capitis (hair shaft and scalp), tinea corporis (body), tinea cruris (groin), tinea pedis (foot), and tinea unguium (hand). Tinea capitis is an infection of the scalp and hair shaft caused by several species of Trichophyton and Microsporum [1, 6-8]. It is the most common dermatophytosis in children aged 
between six months and prepubertal age [8-11]. Tinea capitis is reported more in males than in females within prepubertal age $[7,12]$.

Tinea capitis is quite common in Africa and its prevalence among children is $14-86 \%$ [1]. Studies carried out in different parts of Nigeria have proven that causative agents of tinea capitis vary from one location to another. Nweze [13] reported Trichophyton schoenleinii as the predominant cause of tinea capitis in Borno. In a similar study carried out on 2150 itinerant Quranic scholars in Kano State, Trichophyton rubrum was the most prevalent followed by Microsporum audouinii [14]. Ayanlowo et al. [8] reported Trichophyton mentagrophytes as the most prevalent causative agent in a community in the southwestern part of Nigeria. Nweze and Okafor [15] reported Trichophyton tonsurans as the most prevalent aetiological agent of tinea capitis in Anambra State, Nigeria.

Studies are required to obtain information on its epidemiology and familial, social, and geographical factors $[16,17]$. Although there are studies on prevalence of tinea capitis in different parts of Nigeria, there are no reports on epidemiologic studies on tinea capitis among school children in Kaduna State. Nok community, a rural settlement in Jaba local government area of Kaduna State, was selected for this study. This study was designed to determine the prevalence of tinea capitis and identify factors associated with the infection among primary school children in Nok community, Kaduna State, Nigeria.

\section{Methods}

2.1. Study Area. The study was conducted in Nok community, a rural settlement located in Jaba local government area of Kaduna State, Nigeria (Figure 1). Following the accidental unearthing of Nok terracotta heads (dated to 500 BC) by tin miners in 1943, Nok is one of the most ancient sites of ironwork and terracotta figurine production in Africa [18]. The native language of the people is "hyam." The occupations of the people include farming, trading, and mining. Nok is a typical African settlement with residents having large and extended families living together. The social amenities in the community include electricity, pipe borne water, community health centre, museum, police station, and government primary and secondary schools. Children in the community are enrolled in either of the two universal basic education primary schools.

2.2. Study Population. Ethical approval was obtained from the Kaduna State Ministry of Health and Local Authority of the Universal Basic Education of Jaba local government area, before the commencement of field work. The parents of only one hundred children gave consent for their participation in the study.

2.3. Community Education on Tinea Capitis. Parents and children were enlightened on tinea capitis, purpose of the study, and sample collection procedure in the local hyam language. Information such as the local name for tinea capitis, cause of tinea capitis, and local treatment method was obtained from the people in the community.

2.4. Sample Collection and Questionnaire Administration. Each child was examined in a well-lit room and the scalp was examined for scaly grey patches, lusterless hair strands, and purulent lesions [19]. The site of infection was cleaned with $70 \%$ alcohol and followed by the collection of scalp scrapings from actively growing margins of the lesions using sterile scalpel blades [8]. The samples were transported to the laboratory for culture and identification.

Questionnaires were designed and administered to obtain sociodemographic data such as age, gender, number of children in the family, and information on the sharing of fomites.

2.5. Culture, Identification, and Characterization of Dermatophytes. The scalp scrapings were cultured on Sabouraud's dextrose agar plates with $0.05 \mathrm{~g}$ chloramphenicol and 5\% $\mathrm{NaCl}[20,21]$. The media were prepared according to the manufacturers' instructions and followed by the addition of sodium chloride and $0.05 \mathrm{~g}$ chloramphenicol as described by Weitzman and Summerbell [20]. The samples were inoculated onto the prepared medium and incubated for 3-4 weeks at room temperature and examined for growth weekly [22]. A thin preparation of the fungal culture was made with a drop of lactophenol cotton blue solution on a glass slide, covered with a coverslip and observed under a microscope. Dermatophytes were identified based on macroscopic (growth characteristics and pigmentation) and microscopic morphology (formation of macroconidia and microconidia or other typical elements) as described by David et al. [21].

2.6. Data Analysis. Data were analysed by $\chi^{2}$ test for association using SPSS Statistics 18.0 (SPSS Inc., Chicago, IL, USA), with $p<0.05$ defined as the minimum level of significance to test factors associated with tinea capitis.

\section{Results}

3.1. Level of Awareness on Tinea Capitis in Nok Community. The common name for tinea capitis in Nok community is "shaak shantaan" meaning "spider web." The locals believe that tinea capitis is caused by spiders which urinate on the heads of the children, lay their eggs, and spin their web on the head; hence, this causes the radial spread of the infection. As, at the time of sample collection, the locals did not have any traditional form of treatment for tinea capitis infection, an approach taken in the management of lice is employed for the management of tinea capitis. In serious cases of tinea capitis, the child's hair is shaved to allow the thorough scrubbing of the scalp. Also, his/her clothings are soaked in hot water before washing and the sharing of fomites are avoided.

At the time of the study, the community had only one barbing salon. An interview with salon manager revealed the use of single hair clipper which is treated by spraying methylated spirit on the clipper and using a lighter to flame the clipper after every haircut. 

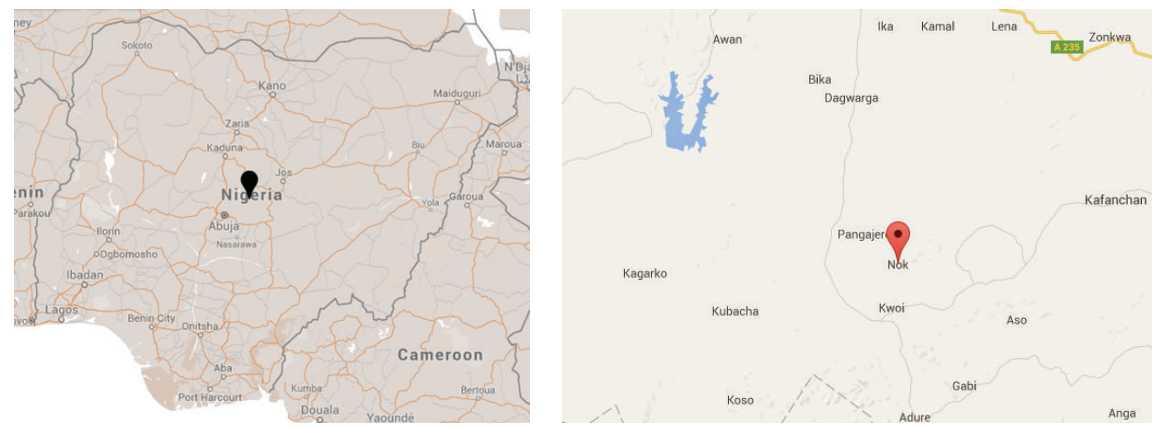

Figure 1: Maps showing Nok village in Kaduna State, Nigeria (taken from Google Maps).

3.2. Sociodemographic Characteristics of Study Subjects. A total of 100 scalp scrapings were collected and analysed for dermatophytes. The characteristics of the study participants are shown in Table 1 . The age range of the study participants was $5-15$ years. Of the 100 participants, $65 \%$ (65/100) were male and $35 \%(35 / 100)$ were female, giving a gender ratio of $1.86: 1$ (males : females). $68 \%$ and $32 \%$ of the participants were of the age range $5-10$ and $11-15$ years, respectively. $74 \%$ of the study participants had a family size of 1-5 members and $26 \%$ of the participants had a family size of 6-10 members. The average number of children per family was 4.3 .

3.3. Prevalence of Tinea Capitis. After the incubation period and examining the microscopic and macroscopic features of fungal growth, there were 45 cases of dermatophytosis of which 33 cases and 12 cases had single and mixed dermatophytes, respectively. The overall prevalence of tinea capitis in the study population is $45 \%$. Tinea capitis was more prevalent among females $(51.4 \% ; 18 / 35)$ than in males $(41.5 \%$; $27 / 65)$. However, the gender ratio in terms of tinea capitis was $1: 1.3$ (male: female) and did not differ significantly $(p=0.402)$. The prevalence of tinea capitis with respect to age was lower for the age group $5-10$ years $(42.6 \% ; 29 / 68)$ than that of $11-$ 15 years $(50 \% ; 16 / 32)$ but was not significantly different $(p=$ $0.524)$. There was an association between tinea capitis and the number of children in a family $(p=0.02)$ and sharing of bed $(p=0.002)$ (Table 1). There was no association between tinea capitis and variables such as sharing of combs $(p=0.188)$, sharing of caps/scarves ( $p=0.107$ ), sharing of towels, hair cut at a barbing salon $(p=0.28)$, keeping and playing with pets $(p=1.00)$, and carrying objects on a bare head $(p=1.00)$.

3.4. Dermatophytes Frequency. Of the 45 cases, a total of 66 dermatophytes were isolated belonging to the genera Microsporum and Trichophyton (Table 2). The most frequently isolated dermatophyte was Trichophyton rubrum (28.8\%; 19/66), followed by Microsporum canis $(22.7 \% ; 15 / 66)$, and the least were Trichophyton verrucosum $(4.5 \% ; 3 / 66)$ and Trichophyton tonsurans $(4.5 \% ; 3 / 66)$. Although 33 cases (73.3\%) had single infections with dermatophytes, mixed infections with 2-4 dermatophytes of the genera Microsporum and Trichophyton were observed in 12 cases (26.7\%). This is presented in relation to age group and gender in Table 3. $75 \%(9 / 12)$ of the mixed infections were with
Microsporum and Trichophyton species, 16.7\% (2/12) were with Microsporum species alone, and $8.3 \%(1 / 12)$ was with Trichophyton species alone.

\section{Discussion}

The high prevalence of tinea capitis (45\%) is close to the prevalence of $43.53 \%$ obtained in a study by Akinboro et al. [19] in Osogbo, southwestern Nigeria. However, the prevalence of this study was higher than those obtained in other studies: 9.5\% for study carried out on 2150 Quranic scholars in Kano State [14], 15.4\% for a study on 604 children in a rural settlement in southwestern Nigeria [8], and 9.4\% for a study in Anambra State involving 47723 children [23]. Moto et al. [24] recently reported a higher prevalence of $81.2 \%$ (122/150) among school children in Mathare, an informal settlement in Nairobi, Kenya. A number of factors may be attributed to the varying prevalence and frequency of tinea capitis in different parts of Nigeria as well as other countries [22]. These include population growth, close contact among infected children at home and school, and poor personal hygiene [24].

With respect to gender, prevalence was higher among females than males, but the association was not significant $(p=0.402)$. This is supported by the findings of some previous studies that reported higher infections among females in Nigeria [25] and Egypt [26].

Furthermore, it was observed that the prevalence of tinea capitis in prepubertal females $(56.7 \% ; 17 / 30)$ was higher than that of the males $(35.3 \% ; 12 / 34)$ while the prevalence of tinea capitis among the pubertal age range of $11-15$ years was higher in males $(48.4 \% ; 15 / 31)$ compared to females $(20 \% ; 1 / 5)$. This is contrary to the findings of Sidat et al. [12] and Balci et al. [7]. This is contrary to some findings and the suggestion that scalp infection in females is less due to steroid-mediated inhibition of dermatophyte growth by progesterone and other similar compounds while males may be predisposed to scalp infection due to prepubertal factors such as level of fungistatic fatty acids [23].

Various conflicting views exist regarding the sexual predominance of tinea capitis which may be attributed to hair dressing and styling practices such as tight hair braiding, shaving of the scalp, plaiting, and the use of hair oils which may promote disease transmission. However, the precise role of such practices remains a subject of study $[3,27,28]$. 
TABLE 1: Demographic data and other factors in relation to tinea capitis among school children in Nok community, Kaduna State, Nigeria.

\begin{tabular}{|c|c|c|c|c|}
\hline Factor & Subcategory & Number of children with tinea capitis & Total number of children & $p$ value \\
\hline \multirow{2}{*}{ Gender } & Male & $27(41.5)$ & 65 & \multirow{2}{*}{0.402} \\
\hline & Female & $18(51.4)$ & 35 & \\
\hline \multirow{2}{*}{ Age group (years) } & $5-10$ & $29(45.3)$ & 64 & \multirow{2}{*}{0.524} \\
\hline & $11-15$ & $16(44.4)$ & 36 & \\
\hline \multirow{2}{*}{ Family size } & $1-5$ & $28(37.8)$ & 74 & \multirow{2}{*}{$0.022^{*}$} \\
\hline & $5-10$ & $17(65.4)$ & 26 & \\
\hline \multirow{4}{*}{ Father's level of education } & None & $2(66.7)$ & 3 & \multirow{4}{*}{0.100} \\
\hline & Primary & $3(30.0)$ & 10 & \\
\hline & Secondary & $31(41.3)$ & 75 & \\
\hline & Tertiary & $9(75.0)$ & 12 & \\
\hline \multirow{4}{*}{ Mother's level of education } & None & $1(100.0)$ & 1 & \multirow{4}{*}{0.414} \\
\hline & Primary & $12(38.7)$ & 31 & \\
\hline & Secondary & $28(45.2)$ & 62 & \\
\hline & Tertiary & $4(66.7)$ & 6 & \\
\hline \multirow{2}{*}{ Awareness about tinea capitis } & Yes & $35(47.3)$ & 74 & \multirow{2}{*}{0.497} \\
\hline & No & $10(38.5)$ & 26 & \\
\hline \multirow{3}{*}{ Hair cut at barbing salons } & Yes & $19(46.3)$ & 41 & \multirow{3}{*}{0.280} \\
\hline & No & $12(35.3)$ & 34 & \\
\hline & No response & $14(56.0)$ & 25 & \\
\hline \multirow{2}{*}{ Sharing beds with other person(s) } & Yes & $36(57.1)$ & 63 & \multirow{2}{*}{$0.002^{*}$} \\
\hline & No & $9(24.3)$ & 37 & \\
\hline \multirow{3}{*}{ Sharing combs with other person(s) } & Yes & $38(48.1)$ & 79 & \multirow{3}{*}{0.188} \\
\hline & No & $6(30.0)$ & 20 & \\
\hline & No response & $1(100.0)$ & 1 & \\
\hline \multirow{2}{*}{ Sharing caps or scarves with other person(s) } & Yes & $23(54.8)$ & 42 & \multirow{2}{*}{0.107} \\
\hline & No & $22(37.9)$ & 58 & \\
\hline \multirow{2}{*}{ Sharing towels with other person(s) } & Yes & $33(47.1)$ & 70 & \multirow{2}{*}{0.661} \\
\hline & No & $12(40.0)$ & 30 & \\
\hline \multirow{2}{*}{ Presence of domestic animals at home } & Yes & $42(44.7)$ & 94 & \multirow{2}{*}{1.00} \\
\hline & No & $3(50.0)$ & 6 & \\
\hline \multirow{2}{*}{ Carrying objects on bare head } & Yes & $41(45.6)$ & 90 & \multirow{2}{*}{1.00} \\
\hline & No & $4(40)$ & 10 & \\
\hline \multirow{3}{*}{ Previous treatment } & Yes & $16(43.2)$ & 37 & \multirow{3}{*}{0.624} \\
\hline & No & $29(46.8)$ & 62 & \\
\hline & No response & $0(0)$ & 1 & \\
\hline
\end{tabular}

* Significantly different ( $p<0.05$ defined as the minimum level of significance).

TABLE 2: Frequency of isolated dermatophytes from school children in Nok community, Kaduna State.

\begin{tabular}{lcc}
\hline Dermatophyte isolated & Frequency & Percentage (\%) \\
\hline Microsporum canis & 15 & 22.7 \\
Microsporum audouinii & 7 & 10.6 \\
Microsporum gypseum & 8 & 12.1 \\
Trichophyton rubrum & 19 & 28.8 \\
Trichophyton mentagrophytes & 4 & 6.1 \\
Trichophyton verrucosum & 3 & 4.5 \\
Trichophyton tonsurans & 3 & 4.5 \\
Trichophyton schoenleinii & 7 & 10.6 \\
\hline
\end{tabular}

The most prevalent dermatophyte isolated was the anthropophilic dermatophyte, Trichophyton rubrum. Other anthropophilic dermatophytes isolated were T. tonsurans, T. schoenleinii, T. rubrum, and M. audouinii. Zoophilic dermatophytes isolated were T. verrucosum and $M$. canis with M. gypseum as the only geophilic dermatophyte isolated. This finding is similar to that of a study carried out in Kano State, Nigeria, by Adeleke et al. [14], where Trichophyton rubrum $(50.2 \%)$ was the most prevalent dermatophyte isolated. Emele and Oyeka [23] reported Microsporum audouinii as the most prevalent dermatophyte (42\%), followed by M. ferrugineum (17\%) and Trichophyton mentagrophytes (16\%) following a study among school children in Anambra State, Nigeria. An aetiological shift with regard to the predominant causative 
TABLE 3: Mixed dermatophyte infection among school children in Nok community, Kaduna State, with respect to age and gender.

\begin{tabular}{|c|c|c|c|}
\hline Case code & Sex & Age group & Dermatophytes isolated \\
\hline NK7 & M & $5-10$ & M. canis, T. rubrum \\
\hline NK15 & $\mathrm{F}$ & $5-10$ & M. gypseum, T. rubrum \\
\hline NK32 & M & $5-10$ & $\begin{array}{l}\text { T. tonsurans, T. schoenleinii, } T \text {. } \\
\text { rubrum }\end{array}$ \\
\hline NK47 & F & $5-10$ & M. canis, M. audouinii \\
\hline NK51 & M & $11-15$ & T. verrucosum, M. canis \\
\hline NK67 & M & $5-10$ & $\begin{array}{l}\text { T. schoenleinii, M. canis, } M \text {. } \\
\text { gypseum }\end{array}$ \\
\hline NK69 & $\mathrm{F}$ & $5-10$ & T. rubrum, M. canis \\
\hline NK72 & M & $5-10$ & $\begin{array}{l}\text { T. schoenleinii, T. rubrum, } M \text {. } \\
\text { canis, M. gypseum }\end{array}$ \\
\hline NK85 & M & $11-15$ & T. schoenleinii, T. rubrum, M. canis \\
\hline NK86 & M & $11-15$ & M. canis, M. audouinii \\
\hline NK90 & M & $11-15$ & $\begin{array}{l}\text { M. audouinii, T. rubrum, T. } \\
\text { tonsurans }\end{array}$ \\
\hline NK94 & $\mathrm{F}$ & $5-10$ & $\begin{array}{l}\text { T. schoenleinii, T. rubrum, } M \text {. } \\
\text { canis, M. gypseum }\end{array}$ \\
\hline
\end{tabular}

M: male; F: female.

agents of tinea capitis has been reported in different parts of the world including the United States, Germany, and parts of Nigeria. This is attributed to factors including the spread of dermatophyte by migrants, change in animal husbandry practices, climate change, and evolution of new genotypes [23].

The finding of $73.3 \%$ single infection and $26.7 \%$ mixed infection with Trichophyton and Microsporum species is supported by $56 \%$ single infection and $38 \%$ mixed infection reported by Moto et al. [24]. However, they reported Epidermophyton species apart from Trichophyton and Microsporum species. They attributed mixed infections to factors such as poor personal hygiene and inadequate treatment. Inadequate treatment may be supported by the data on revealed previous treatment of tinea capitis by $43.2 \%$ of those who had tinea capitis at the time of the study. This is contrary to the finding of Emele and Oyeka [23] and Grover et al. [28] who reported single-dermatophyte infections.

The dominance of anthropophilic dermatophytes may reflect important risk factors involved in the spread of tinea capitis in the community such as direct contact with infected humans or indirect contact with infected fomites [29]. This also may explain the significant association between tinea capitis and the family size and sharing beds. Although $94 \%$ of the children had pets or domestic animals at home and $6 \%$ did not have any pets or domestic animals, there was no significant association with the tinea capitis. Animals such as cats, dogs, rodents, pigs, chickens, cattle, and horses are sources of zoophilic dermatophytes, most often seen on exposed body sites [30,31]. Data regarding the type of animals present in the homes was not collected during the present study.

\section{Conclusion}

Tinea capitis had a prevalence of $45 \%$ among school children in Nok community, Kaduna State, Nigeria. This study showed that the most prevalent causative agents of tinea capitis in Nok community are the anthropophilic dermatophytes, Trichophyton rubrum (28.8\%), followed by the zoophilic dermatophyte, Microsporum canis (22.7\%). Other dermatophytes isolated were Trichophyton mentagrophytes, Trichophyton tonsurans, Trichophyton schoenleinii, Trichophyton verrucosum, Microsporum audouinii, and Microsporum gypseum. These dermatophytes were associated with single and mixed infections, except Trichophyton mentagrophytes, which was not isolated from the cases with mixed infection. There was an association between tinea capitis and the number of family members and sharing of the same bed. This may be attributed to the socioeconomic status of parents and living and sanitary conditions. Hence there is a need to create awareness on tinea infections and preventive measures (such as good personal hygiene and sanitary practice and prompt treatment). There is also a need to educate barbers on the proper disinfection of clippers and the importance of adhering to the procedures. Further studies in different parts of the country would be useful in understanding the epidemiological pattern and management of tinea capitis.

\section{Competing Interests}

The authors declare that they have no competing interests.

\section{References}

[1] R. L. Guerrant, D. H. Walker, and P. F. Weller, Tropical Infectious Diseases: Principles, Pathogens and Practice, Elsevier Churchill, Livingstone, Philadelphia, Pa, USA, 3rd edition, 2011.

[2] L. M. Prescott, J. P. Harley, and D. A. Klein, Microbiology, McGraw Hill, New York, NY, USA, 5th edition, 2002.

[3] E. M. Higgins, L. C. Fuller, and C. H. Smith, "Guidelines for the management of tinea capitis," British Journal of Dermatology, vol. 143, no. 1, pp. 53-58, 2000.

[4] E. I. H. Ménan, O. Zongo-Bonou, F. Rouet et al., “Tinea capitis in schoolchildren from Ivory Coast (western Africa). A 1998-1999 cross-sectional study," International Journal of Dermatology, vol. 41, no. 4, pp. 204-207, 2002.

[5] H. A. Sheikh, "Epidemiology of dermatophytes in the Eastern Province of Saudi Arabia," Research Journal of Microbiology, vol. 4, no. 6, pp. 229-234, 2009.

[6] A. Bennassar and G. Ramon, "Management of tinea capitis in childhood," Clinical, Cosmetic and Investigational Dermatology, vol. 3, pp. 89-98, 2010.

[7] E. Balci, M. Gulgun, O. Babacan et al., "Prevalence and risk factors of tinea capitis and tinea pedis in school children in Turkey," Journal of the Pakistan Medical Association, vol. 64, no. 5, pp. 514-518, 2014.

[8] O. Ayanlowo, A. Akinkugbe, R. Oladele, and M. Balogun, "Prevalence of Tinea capitis infection among primary school 
children in a rural setting in south-west Nigeria," Journal of Public Health in Africa, vol. 5, no. 1, 2014.

[9] M. Feuilhade and C. Lacroix, "Epidemiology of tinea capitis," Presse Medicale, vol. 30, no. 1, pp. 499-504, 2001.

[10] V. Mane, A. D. Urheka, S. Mali, N. Patil, S. A. Patil, and K. G. Ajit, "Tinea capitis infection in children along with tertiary care hospitals with reference to in vitro antifungal susceptibility testing of dermatophytes isolate," International Journal of Research and Reviews in Pharmacy and Applied Science, vol. 3, pp. 199-208, 2013.

[11] M. Mayowa, R. Godson, and A. M. K. Sridhar, "Use of Azadirachta indica derived germicidal in the management of tinea capitis among pupils in selected public primary schools in Ibadan, Nigeria," Peak Journal of Medicinal Plant Research, vol. 3, no. 1, pp. 9-15, 2015.

[12] M. M. Sidat, D. Correia, and T. P. Buene, “Tinea capitis among children at one suburban primary school in the City of Maputo, Mozambique," Revista da Sociedade Brasileira de Medicina Tropical, vol. 40, no. 4, pp. 473-475, 2007.

[13] E. I. Nweze, "Etiology of dermatophytoses amongst children in northeastern Nigeria," Medical Mycology, vol. 39, no. 2, pp. 181$184,2001$.

[14] S. Adeleke, B. Usman, and G. Ihesiulor, "Dermatophytosis among itinerant quranic scholars in Kano (Northwest) Nigeria," Nigerian Medical Practitioner, vol. 53, no. 3, pp. 33-35, 2008.

[15] E. I. Nweze and J. I. Okafor, "Prevalence of dermatophytic fungal infections in children: a recent study in Anambra State, Nigeria," Mycopathologia, vol. 160, no. 3, pp. 239-243, 2005.

[16] I. J. Frieden and R. Howard, "Tinea capitis: epidemiology, diagnosis, treatment, and control," Journal of the American Academy of Dermatology, vol. 31, no. 3, part 2, pp. S42-S46, 1994.

[17] A. Yazdanfar, "Tinea capitis in primary school children in Hamedan (west of Iran)," International Journal of Medicine and Medical Sciences, vol. 2, pp. 29-33, 2010.

[18] Department of Arts of Africa, Oceania, and The Americas, "Nok Terracottas (500 B.C.-200 A.D.)," in Heilbrunn Timeline of Art History, The Metropolitan Museum of Art, New York, NY, USA, 2000, http://www.metmuseum.org/toah/hd/nok/hd_nok.htm.

[19] O. Akinboro, A. Olayinka, A. Olasode, and O. Onayemi, "The pattern, Risk factors and clinico-aetiological correlate of tinea capitis among the children in a tropical community setting of Osogbo, South-Western Nigeria," South-Western Nigeria. AfroEgypt Journal of Endemic Diseases, vol. 1, no. 2, pp. 53-64, 2011.

[20] I. Weitzman and R. C. Summerbell, "The dermatophytes," Clinical Microbiology Reviews, vol. 8, no. 2, pp. 240-259, 1995.

[21] E. David, S. Davis, H. Alexiou, R. Handke, and R. Bartley, Description of Medical Fungi, The Authors, Adelaide, Australia, 2nd edition, 2007.

[22] I. Ahmed, A. Zaffar, and N. Sarwat, "Prevalence of tinea capitis and asymptomatic carriage amongst school going children," Journal of Pakistan Association of Dermatologists, vol. 16, pp. 215-219, 2006.

[23] F. E. Emele and C. A. Oyeka, "Tinea capitis among primary school children in Anambra state of Nigeria," Mycoses, vol. 51, no. 6, pp. 536-541, 2008.

[24] J. N. Moto, J. M. Maingi, and A. K. Nyamache, "Prevalence of Tinea capitis in school going children from Mathare, informal settlement in Nairobi, Kenya," BMC Research Notes, vol. 8, article 274, 2015.

[25] J. C. Anosike, I. R. Keke, J. C. Uwaezuoke et al., "Prevalence and distribution of ringworm infection in primary schools in parts of Eastern Nigeria," Journal of Applied Sciences and Environmental Management, vol. 9, no. 3, pp. 21-25, 2006.

[26] A. A. Omar, "Ringworm of the scalp in primary-school children in Alexandria: infection and carriage," Eastern Mediterranean Health Journal, vol. 6, no. 5-6, pp. 961-967, 2000.

[27] S. F. Friedlander, M. Rueda, B. K. Chen, and H. W. Caceros-Rios, "Fungal, protozoal and helminthic infections," in Paediatric Dermatology, L. A. Schachner and R. C. Hansen, Eds., pp. 10931140, Mosby, St. Louis, Mo, USA, 3rd edition, 2003.

[28] C. Grover, P. Arora, and V. Manchanda, "Tinea capitis in the pediatric population: a study from North India," Indian Journal of Dermatology, Venereology and Leprology, vol. 76, no. 5, pp. 527-532, 2010.

[29] L. Johnson, "Dermatophytes-the skin eaters," Mycologist, vol. 17, no. 4, pp. 147-149, 2003.

[30] P. M. Rabinowitz, Z. Gordon, and L. Odofin, "Pet-related infections," American Family Physician, vol. 76, no. 9, pp. 13141322, 2007.

[31] B. D. Michaels and J. Q. Del Rosso, "Tinea capitis in infants: recognition, evaluation, and management suggestions," The Journal of Clinical and Aesthetic Dermatology, vol. 5, no. 2, pp. 49-59, 2012. 


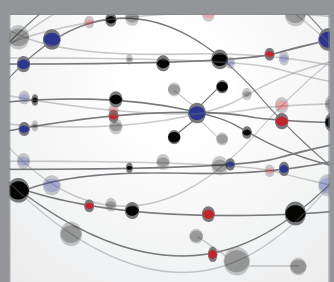

The Scientific World Journal
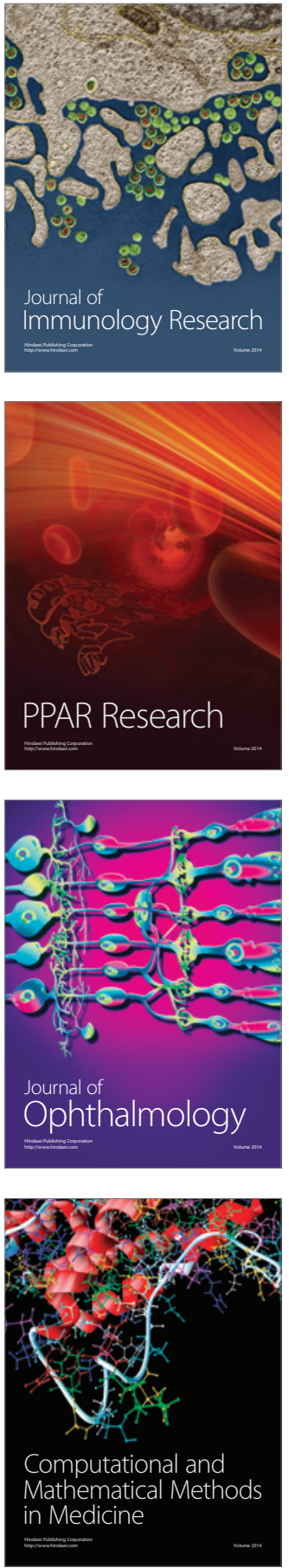

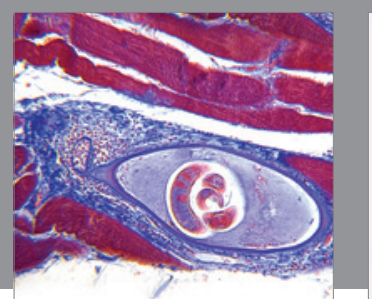

Gastroenterology Research and Practice

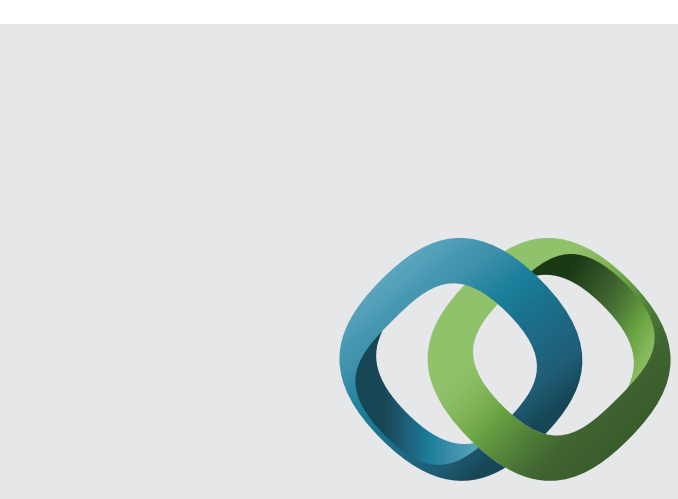

\section{Hindawi}

Submit your manuscripts at

http://www.hindawi.com
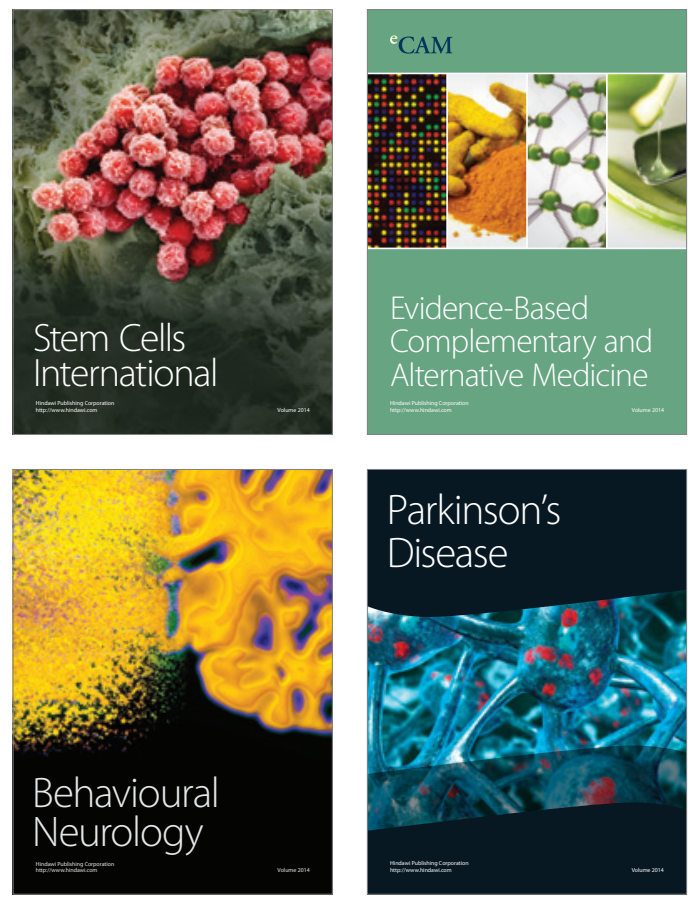
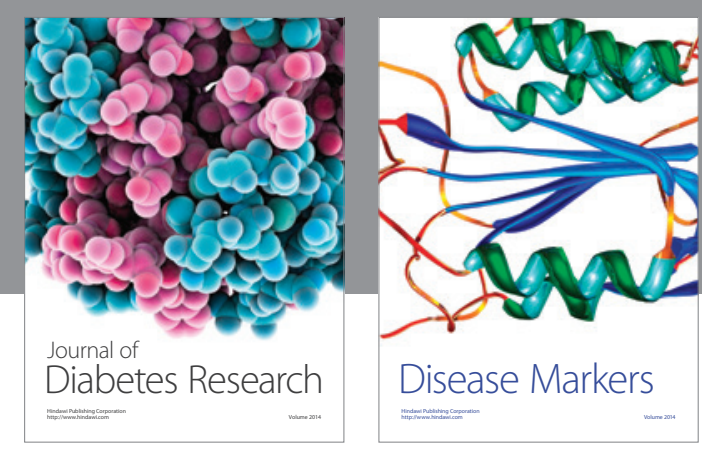

Disease Markers
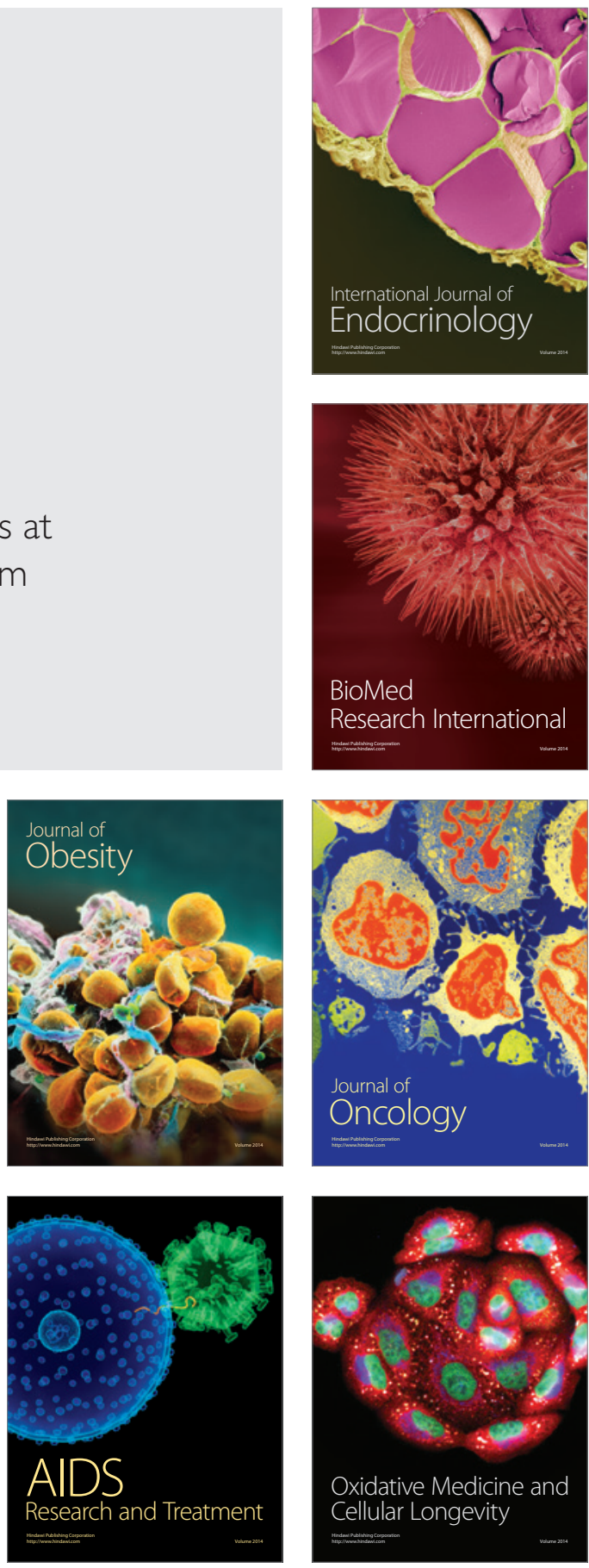